\title{
ADHERENT COMPACT SPACES
}

\author{
ROBERT L. KRYSTOCK
}

(Communicated by James E. West)

\begin{abstract}
A new class of $H$-closed spaces, herein referred to as the class of adherent compact spaces, is introduced-and shown to be properly contained in the class of $C$-compact spaces. It is also shown, using a construction of Goss and Viglino, that these spaces need not be seminormal.
\end{abstract}

\section{INTRODUCTION}

$H$-closed spaces were introduced by Alexandroff and Urysohn at a time when our present-day definition of compactness had not yet been settled upon. The term "compact" had been used in reference to sequential compactness, and in reference to countable compactness. It had not yet been determined that compactness, as defined today, is preserved in the passage to products; and, Alexandroff and Urysohn had only recently applied our present-day definition of compactness to topological spaces in general. (See Willard [13], Historical Notes.)

A space $X$ is $H$-closed (Hausdorff-closed) if $X$ is a closed subset of every space in which $X$ is embedded. (All the spaces considered herein are assumed to be Hausdorff.) Equivalently, $X$ is $H$-closed if and only if (i) every open cover of $X$ contains a finite subcollection whose closures cover $X$, or (ii) every open filter on $X$ has nonempty adherence.

Whereas compactness is an absolute property, $H$-closure is not. An $H$-closed space is necessarily $H$-closed relative to every space in which it is embedded, but a subset of a space $Y$ which is $H$-closed relative to $Y$ need not be $\mathrm{H}$ closed. (A subset $S$ of a space $Y$ is $H$-closed relative to $Y$ if every cover of $S$ consisting of open subsets of $Y$ contains a finite subcollection whose closures in $Y$ cover $X$.) On the other hand, whereas a space $X$ can be embedded in a compact space if and only if it is Tychonoff, every space $X$ can be embedded in an $H$-closed space. (See Katětov [6], Fomin [3], and Liu [8].)

Received by the editors April 18, 1988 and, in revised form, January 23, 1989. Presented to the American Mathematical Society at the 94th Annual Meeting (Joint AMS-MAA Meetings) on January 6-9, 1988 in Atlanta, Georgia.

1980 Mathematics Subject Classification (1985 Revision). Primary 54D25.

$K e y$ ' words and phrases. $H$-closed, $H$-set, $C$-compact, seminormal, open filter adherence. 
A subset of a space $Y$ which is $H$-closed relative to $Y$ is called an $H$-set (of $Y$ ); and, although $H$-sets have thus far played an important role in the development of the theory of $H$-closed spaces, the exact relationship between $H$-sets and $H$-closed subspaces is as yet undetermined. There is evidence that, to some extent, the two are similar, e.g., whereas the cardinality inequalities for the number of compact subspaces of a space (established by Burke and Hodel in [2]) are satisfied when the words "compact subspaces" are replaced by " $\mathrm{H}$ closed subspaces," they are also satisfied when the words "compact subspaces" are replaced by " $H$-sets" (Bella [1]). On the other hand, there is evidence that the difference between the two is vast, e.g., a space in which every closed subset is $H$-closed is necessarily a compact space (M. H. Stone [10] and Katětov [6]), but a space in which every closed subset is an $H$-set need not be compact (Viglino [11]).

That difficulties have been encountered in dealing with $H$-sets is evidenced by the situation regarding the $C$-compact spaces (those $H$-closed spaces having the property that every closed subset is an $H$-set) and the $H$-closed seminormal spaces (those $H$-closed spaces having the property that every closed subset possesses a neighborhood base consisting of regular open sets). Whereas, for the past eighteen years, the $H$-closed seminormal spaces have comprised the only known class of noncompact $H$-closed spaces properly contained in the class of $C$-compact spaces, little is known regarding either of these classes of spaces. (Although it is well known that every continuous mapping of a $C$ compact space (into a Hausdorff space) is a closed mapping, this property is not a characterization of $C$-compact spaces (Goss-Viglino [4]). As in the case of the $C$-compact spaces, the $H$-closed seminormal spaces can be defined solely in terms of $H$-sets (see Krystock [7]).

It was previously thought that further development in the area of $C$-compact spaces would have to await the discovery of new characterizations of $H$-setscharacterizations which would render the $H$-sets easier to deal with (if indeed such characterizations exist). However, the results obtained herein provide for an alternative. Previously, this author showed in [7] that although an $H$-set of an $H$-closed space $X$ need not be the adherence of some open filter defined on $X$, the open filter adherences of $X$ are necessarily $H$-sets of $X$. More recently, Porter and Tikoo showed in [9] that every $H$-closed subspace of an $H$-closed space is an open filter adherence. Moreover, the $H$-closed space $h W$ of Example 2.4 of [7] was therein shown to have the property that every $H$-set of $h W$ is the adherence of some open filter defined on $h W$, and it is well known that $h W$ contains an $H$-set which is not $H$-closed. Thus, in an $H$ closed space, every $H$-closed subspace is an open filter adherence, every open filter adherence is an $H$-set, and neither of these implications reverses. For the first time, there is a concept which separates $H$-sets and $H$-closed subspacessuggesting (in view of the results obtained by Stone and Viglino) the existence of noncompact $C$-compact spaces which are not defined in terms of $H$-sets in general. This existence will be established below. 
In what follows, attention will be focused upon those $H$-closed spaces having the property that every closed subset is an open filter adherence. These spaces will herein be called adherent compact spaces, and will be shown in $\S 3$ to comprise a class of $H$-closed spaces which is properly contained between the class of compact spaces and the class of $C$-compact spaces. In $\S 4$, it will be shown that adherent compact spaces need not be seminormal, thus establishing that the adherent compact spaces comprise a previously unknown class of $H$-closed spaces.

\section{BASIC DEFINITIONS AND NOTATION}

A topological space $X$ is $C$-compact if every closed subset of $X$ is an $H$-set (of $X$ ). A nonempty subset $S$ of a space $X$ is an $H$-set (of $X$ ) if every open filter on $X$ which meets $S$, has a cluster point in $S$; and $X$ is $H$-closed if $X$ is an $H$-set of $X$.

If $\mathscr{F}$ is an open filter on a space $X$ and $S \subseteq X$, then $\mathscr{F}$ meets $S$ if each $F \in \mathscr{F}$ meets $S$ (i.e., if $F \cap S \neq \phi$ for each $F \in \mathscr{F}$ ); and $x \in X$ is a cluster point of $\mathscr{F}$ if $x \in$ ad $\mathscr{F} \equiv \cap_{F \in \mathcal{F}} \mathrm{cl} F$. A subset $S \subseteq X$ is an open filter adherence if $S=\operatorname{ad} \mathscr{G}$ for some open filter $\mathscr{G}$ on $X$.

A space $X$ is seminormal if every closed subset of $X$ possesses a neighborhood base consisting of regular open sets, and $S \subseteq X$ is regular open if $S=\operatorname{int}(\operatorname{cl} S)$.

For a subset $S$ of a space $X, \eta_{S}$ will be used to denote the open neighborhood filter of $S$; and if $S=\{x\}, \eta_{x}$ will be used. The $\theta$-closure of $S$ is the set $\operatorname{cl}_{\theta} S \equiv\left\{x \in X \mid \operatorname{cl} N \cap S \neq \phi\right.$ for all $\left.N \in \eta_{x}\right\}$.

\section{C-COMPACTNESS AND OPEN FILTER ADHERENCES}

Example 3.1 [11, Example 2]. Let $A \equiv\left\{a_{i j}=(1 / i, 1 / j) \mid i, j \in N\right\}$ and $C \equiv$ $\left\{c_{i}=(1 / i, 0) \mid i \in N\right\}$ where $N$ denotes the set of positive integers. Let $X=$ $A \cup C \cup\{\alpha\}$, and let $\left\{N_{i}\right\}_{i \in N}$ be a partition of $N$ into infinitely many infinite equivalence classes. The topology on $A$ is the discrete topology, and a basic neighborhood of $c_{i}($ resp. $\alpha)$ is of the form

$$
\begin{aligned}
& U_{k}\left(c_{i}\right) \equiv\left\{c_{i}\right\} \cup\left\{a_{i j} \mid j \geq k\right\} \cup\left\{a_{p j} \mid p \geq k, j \in N_{i}\right\} \\
& \text { (resp. } U_{k, F}(\alpha) \equiv\{\alpha\} \cup\left\{a_{p j} \mid p \geq k, j \notin \bigcup_{i \in F} N_{i}\right\}
\end{aligned}
$$

where $F$ is any finite subset of $N$ ). The space $X$ is $H$-closed and seminormal. (See [12].)

Remark 3.2. The point $\alpha \in X$ of Example 3.1 is denoted by $\infty$ in Example 2 of [11]; and although the neighborhoods of $\infty$ were therein defined in less restrictive fashion, it is easily seen that they yield the same neighborhood system as do the $U_{k, F}(\alpha)$ 's. 
Remark 3.3. Note that for fixed $n \in N$, the assignment to each $c_{i}$ (resp. to $\alpha$ ), basic neighborhoods of the form

$$
\begin{aligned}
& U_{n, k}\left(c_{i}\right) \equiv\left\{c_{i}\right\} \cup\left\{a_{i j} \mid j \geq k\right\} \cup\left\{a_{p j} \mid p \geq k, j \in N_{i+n}\right\} \\
& \text { resp. } U_{n, k, F}(\alpha) \equiv\{\alpha\} \cup\left\{a_{p j} \mid p \geq k, j \notin \bigcup_{i \in F} N_{i+n}\right\}
\end{aligned}
$$

yields an $H$-closed seminormal space-which will be denoted by $X_{n}$. (Easily, $X_{n}$ is $H$-closed; and, seminormality follows directly from the argument given in Example 2 of [12].)

Example 3.4. For $n \in N$, and a fixed partition $\left\{N_{i}\right\}_{i \in N}$, let $X_{n}$ denote the $H$-closed seminormal space described in Remark 3.3; and for $n=\infty$, let $X_{n}$ denote a copy of $X_{1}$. For $n \in N^{*} \equiv N \cup\{\infty\}, A_{n}$ (resp. $C_{n}$ ) will be used to denote the copy of $A$ (resp. $C$ ) in $X_{n}$. Correspondingly, $c_{i}^{n}$ (resp. $a_{i j}^{n}, \alpha^{n}$ ) will denote the point $c_{i}$ (resp. $\left.a_{i j}, \alpha\right)$ of $X_{n}$.

Let $W$ be the quotient space obtained from the disjoint union, $\bigcup_{n \in N^{*}} X_{n}$, by identifying $c_{i}^{\infty}$ with $\alpha^{i}$ for each $i \in N$. Let $\phi$ be a bijection of $\bigcup_{n \in N} C_{n}$ onto $N$, and let $Y$ denote the space consisting of all points in $W \cup\{\beta\}$ with topology determined as follows:

for $y \notin\{\beta\} \cup\left(\cup_{n \in N} C_{n}\right)$, the basic neighborhoods of $y$ are as defined in $W$,

for $c_{i}^{m} \in \bigcup_{n \in N} C_{n}$, the basic neighborhoods of $c_{i}^{m}$ are of the form $N_{k}\left(c_{i}^{m}\right)=U_{m, k}\left(c_{i}^{m}\right) \cup V_{k}\left(c_{i}^{m}\right)$ where $U_{m, k}\left(c_{i}^{m}\right)$ is as defined in $X_{m}$, and $V_{k}\left(c_{i}^{m}\right)=$ $\left\{a_{p j}^{n} \mid p \in N, j \in N_{\phi\left(c_{i}^{m}\right)}, k \leq n<\infty\right\}$, and

a basic neighborhood of $\beta$ is of the form $N_{k, F}(\beta)=\{\beta\} \cup V_{k, F}(\beta)$ where $V_{k, F}(\beta)=\left\{a_{p j}^{n} \mid p \in N, j \notin \bigcup_{c_{i}^{m} \in F} N_{\phi\left(c_{i}^{m}\right)}, k \leq n<\infty\right\}$ and $F$ is any finite subset of $\bigcup_{n \in N} C_{n}$.

Note that for $i, m$, and $k$, in $N$, and each $F \subseteq \bigcup_{n \in N} C_{n}, \bigcup_{k \leq n<\infty} C_{n} \subseteq$ $\operatorname{cl} N_{k}\left(c_{i}^{m}\right) \cap \operatorname{cl} N_{k, F}(\beta)$.

\section{Proposition 3.5. The space $Y$ is C-compact.}

Proof. $W$ is Hausdorff by construction; and it is easily seen that $\left\{V_{k}\left(c_{i}^{m}\right) \mid k \in\right.$ $N\}$ and $\left\{V_{k, F}(\beta) \mid k \in N, F\right.$ is a finite subset of $\left.\bigcup_{n \in N} C_{n}\right\}$ are bases for free open filters on $Y \backslash\left[\{\beta\} \cup\left(\bigcup_{n \in N} C_{n}\right)\right]$. Thus, to show that $Y$ is Hausdorff, it suffices to show that each pair of points in $\{\beta\} \cup\left(\cup_{n \in N} C_{n}\right)$ can be separated by disjoint open sets. So let $c_{i}^{s}, c_{j}^{r} \in \cup_{n \in N} C_{n}$ with $s \neq r$ and/or $i \neq j$, and let $m=\max \{i+j, r+s\}$. Then $N_{m}\left(c_{i}^{s}\right), N_{m}\left(c_{j}^{r}\right)$, and $N_{m, F}(\beta)$, where $F=\left\{c_{i}^{s}, c_{j}^{r}\right\}$ are pairwise disjoint basic neighborhoods. Thus $Y$ is Hausdorff.

Now let $B$ be any closed subset of $Y$; and let $\mathscr{G}$ be any open filter on $Y$ which mets $B$. If $\mathscr{G}$ meets $B \cap X_{n}$ for some $n \in N^{*}$, then $\mathscr{G} \cap X_{n} \equiv$ $\left\{G \cap X_{n} \mid G \in \mathscr{G}\right\}$ is a base for an open filter on $X_{n}$ which meets the closed subset $B \cap X_{n}$ of $X_{n}$. Since $X_{n}$ is $C$-compact, $\mathscr{G} \cap X_{n}$, and therefore $\mathscr{G}$, 
has a cluster point in $B \cap X_{n} \subseteq B$. On the other hand, if $\mathscr{G}$ fails to meet each $B \cap X_{n}$, then for $k \in N$ there exists $G_{k} \in \mathscr{G}$ such that $G_{k} \cap B \subseteq$ $\bigcup_{k \leq n<\infty} X_{n} \backslash\left\{\alpha^{n}\right\}$; and if $G_{k} \cap B \cap\left(\bigcup_{n \in n} C_{n}\right) \neq \phi$ for $k \in N$, then for $G \in \mathscr{G}$, $\phi \neq G \cap G_{k} \cap B \cap\left(\bigcup_{n \in N} C_{n}\right) \subseteq G \cap G_{k} \cap B \cap\left(\bigcup_{k \leq n<\infty} C_{n}\right) \subseteq \operatorname{cl} N_{k}\left(c_{i}^{m}\right)$ for every $c_{i}^{m} \in \bigcup_{n \in N} C_{n}$ and therefore for every $c_{i}^{m} \in B \cap\left(\bigcup_{n \in N} C_{n}\right)$. It follows that for $c_{i}^{m} \in B \cap\left(\bigcup_{n \in N} C_{n}\right), N_{k}\left(c_{i}^{m}\right) \in \eta_{c_{i}^{m}}$ and $G \in \mathscr{G}, G \cap N_{k}\left(c_{i}^{m}\right) \neq \phi$ so that $\mathscr{G}$ has a cluster point in $B \cap\left(\bigcup_{n \in N} C_{n}\right) \subseteq B$.

Now suppose that $\mathscr{G}$ fails to meet each $B \cap X_{n}, n \in N^{*}$, and that $\widetilde{G} \cap$ $B \cap\left(\bigcup_{n \in N} C_{n}\right)=\phi$ for some $\widetilde{G} \in \mathscr{G}$. If for $c_{i}^{m} \in \bigcup_{n \in N} C_{n}, c_{i}^{m} \notin \operatorname{ad} \mathscr{G} \cap B$, let $N_{k, F}(\beta)=\{\beta\} \cup\left\{a_{p q}^{n} \mid p \in N, q \notin \bigcup_{c_{i}^{m} \in F} N_{\phi\left(c_{i}^{m}\right)}, k \leq n<\infty\right\}$ be any basic neighborhood of $\beta$, and let $G$ be any element of $\mathscr{G}$. There exists $\widehat{G} \in \mathscr{G}$ and $s \in N$ such that $N_{s}\left(c_{i}^{m}\right) \cap \widehat{G} \cap B=\phi$ for each $c_{i}^{m} \in F$ so that if $r=\max \{k, s\}$, then $\bar{G} \equiv G_{r} \cap \widetilde{G} \cap \widetilde{G} \cap G \in \mathscr{G}$ such that $\phi \neq \bar{G} \cap B \subseteq G \cap B \cap\left[\bigcup_{r \leq n<\infty} X_{n} \backslash\left\{\alpha^{n}\right\}\right] \cap$ $\left[Y \backslash \bigcup_{n \in N} C_{n}\right] \cap\left[Y \backslash \bigcup_{c_{i}^{m} \in F} N_{r}\left(c_{i}^{m}\right)\right]=G \cap B \cap\left\{a_{p q}^{n} \mid p \in N, q \notin \bigcup_{c_{i}^{m} \in F} N_{\phi\left(c_{i}^{m}\right)}, r \leq\right.$ $n<\infty\} \subseteq N_{k, F}(\beta)$. 'It follows that $\beta \in(\operatorname{ad} G) \cap(\operatorname{cl} B)=(\operatorname{ad} G) \cap B$, and therefore, $Y$ is $C$-compact.

The next lemma follows directly from the proof of Proposition (2.3) of [7].

Lemma 3.6. A nonempty subset $S$ of an $H$-closed space $X$ is an open filter adherence if and only if, for $x \in \mathrm{cl}_{\theta} S \backslash S$, there exists $N_{x} \in \eta_{x}$ such that for every finite subset $\left\{x_{1}, x_{2}, \ldots, x_{n}\right\} \subseteq \operatorname{cl}_{\theta} S \backslash S, \operatorname{int}\left(\bigcup_{i=1}^{n} \operatorname{cl} N_{x_{i}}\right) \cap S=\phi$.

Proposition 3.7. The subset, $\cup_{n \in N} C_{n}$, of $Y$ is a closed subset of $Y$ which is not an open filter adherence.

Proof. It is easy to see that $\bigcup_{n \in N} C_{n}$ is closed and that $\mathrm{cl}_{\theta}\left(\bigcup_{n \in N} C_{n}\right) \backslash \bigcup_{n \in N} C_{n}$ $=\{\beta\} \cup\left\{\alpha^{i} \mid i \in N\right\}$. Let $\left\{N_{k, F}(\beta)\right\} \cup\left\{U_{i, k_{i}, F_{i}}\left(\alpha^{i}\right) \mid i \in N\right\}$ be any cover of $\mathrm{cl}_{\theta}\left(\bigcup_{n \in N} C_{n}\right) \backslash \bigcup_{n \in N} C_{n}$ by basic neighborhoods. Let $r>\max \left(\{k\} \cup\left\{m \mid c_{i}^{m} \in\right.\right.$ $\left.F\} \cup\left\{\phi\left(c_{i}^{m}\right) \mid c_{i}^{m} \in F\right\}\right)$ and let $t>\max \left\{r, k_{r}\right\}$. It will be shown that $N_{r}\left(c_{t}^{r}\right) \subseteq$ $\operatorname{cl} N_{k, F}(\beta) \cup \operatorname{cl} U_{r, k_{r}, F_{r}}\left(\alpha^{r}\right)$. Now

(i) $t>r>\max \left(\{k\} \cup\left\{m \mid c_{i}^{m} \in F\right\}\right)$ implies that $c_{t}^{r} \in \operatorname{cl} N_{k, F}(\beta)$ and $c_{t}^{r} \notin F$ so that $V_{r}\left(c_{t}^{r}\right)=\left\{a_{p j}^{n} \mid p \in N, j \in N_{\phi\left(c^{r}\right)}, n \geq r\right\} \subseteq\left\{a_{p j}^{n} \mid p \in\right.$ $\left.N, j \notin \bigcup_{c_{i}^{\prime \prime} \in F} N_{\phi\left(c_{i}^{\prime \prime}\right)}, n \geq r\right\}=V_{t, F}(\beta) \subseteq V_{k, F}(\beta) \subseteq N_{k, F}(\beta)$,

(ii) $t>r>\max \left(\{k\} \cup\left\{\phi\left(c_{i}^{m}\right) \mid c_{i}^{m} \in F\right\}\right)$ implies that $\left\{a_{p j}^{r} \mid p \geq r, j \in N_{t+r}\right\} \subseteq$ $\left\{a_{p j}^{n} \mid p \in N, j \notin \bigcup_{c_{i}^{m} \in F} N_{\phi\left(c_{i}^{m}\right)}, n \geq r\right\}=V_{r, F}(\beta) \subseteq V_{k, F}(\beta) \subseteq N_{k, F}(\beta)$, and

(iii) $t>\max \left\{r, k_{r}\right\}$ and $r>\max \left(\{k\} \cup\left\{\phi\left(c_{i}^{m}\right) \mid c_{i}^{m} \in F\right\}\right)$ imply $\left\{a_{t j}^{r} \mid j \geq\right.$ $r\}=\left\{a_{t j}^{r} \mid j \notin \bigcup_{c_{i}^{m} \in F} N_{\phi\left(c_{i}^{m}\right)}, j \geq r\right\} \cup\left\{a_{t j}^{r} \mid j \in \bigcup_{c_{i}^{m} \in F} N_{\phi\left(c_{i}^{m}\right)}, j \geq r\right\} \subseteq$ $V_{r, F}(\beta) \cup\left\{a_{p j}^{r} \mid p \geq t, j \notin \bigcup_{i \in F_{r}} N_{i+r}\right\} \subseteq N_{r, F}(\beta) \cup\left\{a_{p j}^{r} \mid p \geq k_{r}, j \notin\right.$ $\left.\bigcup_{i \in F_{r}} N_{i+r}\right\} \subseteq N_{k, F}(\beta) \cup U_{r, k_{r}, F_{r}}\left(\alpha^{r}\right)$. 
Thus $N_{r}\left(c_{t}^{r}\right)=U_{r, r}\left(c_{t}^{r}\right) \cup V_{r}\left(c_{t}^{r}\right)=\left\{c_{t}^{r}\right\} \cup\left\{a_{t j}^{r} \mid j \geq r\right\} \cup\left\{a_{p j}^{r} \mid p \geq r, j \in N_{t+r}\right\} \cup$ $V_{r}\left(c_{t}^{r}\right) \subseteq\left\{c_{t}^{r}\right\} \cup\left\{a_{t j}^{r} \mid j \geq r\right\} \cup N_{k, F}(\beta) \subseteq\left\{c_{t}^{r}\right\} \cup U_{r, k_{r}, F_{r}}\left(\alpha^{r}\right) \cup N_{k, F}(\beta) \subseteq \operatorname{cl} N_{k, F}(\beta) \cup$ $\operatorname{cl} U_{r, k_{r}, F_{r}}\left(\alpha^{r}\right)$ so that $\bigcup_{n \in N} C_{n}$ is not an open filter adherence.

Remark 3.8. Note that any open cover of $\bigcup_{n \in N} C_{n}$ by basic neighborhoods contains no finite subcover, so that $\bigcup_{n \in N} C_{n}$ is not a compact subset of $Y$. Thus $Y$ is not compact.

Lemma 3.9. [7, Proposition (2.2)]. If $S$ is an open filter adherence of an $\mathrm{H}$ closed space $X$, then $S$ is an $H$-set of $X$.

Proposition 3.10. If $X$ is $H$-closed and every closed subset of $X$ is an open filter adherence, then $X$ is $C$-compact.

Proof. This follows directly from Lemma 3.9.

Definition 3.11. A space $X$ will be called adherent compact if $X$ is $H$-closed and every closed subset of $X$ is an open filter adherence.,

Corollary 3.12. The class of adherent compact spaces is properly contained between the class of compact Hausdorff spaces and the class of C-compact spaces.

\section{AdHERENT COMPACT SPACES NEED NOT BE SEMINORMAL}

Example 4.1. [5, Example]. Let $A \equiv\left\{a_{n m}=(1 / 2 n-1,1 / m) \mid n, m \in N\right\}$, $B \equiv\left\{b_{n m}=(1 / 2 n,-1 / m) \mid n, m \in N\right\}, C \equiv\left\{c_{n}=(1 / 2 n-1,0) \mid n \in N\right\}$, and $D \equiv\left\{d_{n}=(1 / 2 n, 0) \mid n \in N\right\}$. Let $\left\{N_{j}\right\}_{j \in N}$ (resp. $\left\{N_{1}^{i}\right\}_{i \in N}$ ) be a partition of $N$ (resp. $N_{1}$ ) into infinitely many infinite equivalence classes, and let $\phi$ be a bijection of $A$ to $N \backslash\{1\}$. Let $X=A \cup B \cup C \cup D \cup\{\infty\}$ and, for $x \in X$, let a basic neighborhood $N_{x}$ of $x$ be determined as follows:

if $x \in A$, then $N_{x}$ is of the form $N_{k}\left(a_{i j}\right) \equiv\left\{a_{i j}\right\} \cup\left\{b_{n m} \mid n \geq k, m \in\right.$ $\left.N_{\phi\left(a_{i j}\right)}\right\}$ where $k \in N$;

if $x \in B$, then $N_{x} \equiv\{x\}$; i.e., $B$ is discrete;

if $x \in C$, then $N_{x}$ is of the form $N_{k}\left(c_{i}\right) \equiv\left\{c_{i}\right\} \cup H \cup\left\{b_{p q} \mid p \geq k, q \in\right.$ $\left.\bigcup_{a \in H} N_{\phi(a}\right\}$ where $H \equiv\left\{a_{i m} \mid m \geq k\right\} \cup\left\{a_{n m} \mid n \geq k, m \in N_{i}\right\}$ and $k \in N$;

if $x \in D$, then $N_{x}$ has the form $N_{k}\left(d_{i}\right) \equiv\left\{d_{i}\right\} \cup\left\{b_{i j} \mid j \geq k\right\} \cup\left\{b_{n m} \mid n \geq\right.$ $\left.k, m \in N_{1}^{i}\right\}$ where $k \in N$; and

if $x=\infty$, then $N_{x}$ is of the form $N_{k, r}(\infty) \equiv\{\infty\} \cup\left(X \backslash T_{k, r}\right)$ where $T_{k, r}=C \cup D \cup\left(\bigcup_{i=1}^{k}\left[N_{r}\left(c_{i}\right) \cup N_{r}\left(d_{i}\right)\right]\right)$ and $k, r \in N$.

$X$ is $C$-compact but not seminormal.

Remark 4.2. It is easily seen that the assignment of neighborhoods to points in $X \backslash\{\infty\}$ is a valid one. And, Lemma 4.4 will yield $\operatorname{cl} T_{k, r}=C \cup D \cup$ $\left(\bigcup_{i=1}^{k}\left[\operatorname{cl} N_{r}\left(c_{i}\right) \cup \operatorname{cl} N_{r}\left(d_{i}\right)\right]\right)=T_{k, r}$ so that the assignment above is valid for all points in $X$.

Remark 4.3. The space $X$ of Example 4.1 is essentially the space constructed by Goss and Viglino in [5]. Because of misprints it is difficult to ascertain 
precisely how the basic neighborhoods were defined therein. However, with the basic neighborhoods defined as in Example 4.1, it is easily seen that $X$ is $H$-closed and that the argument of [5] regarding seminormality remains valid. $C$-compactness of $X$ will follow from Propositions 4.5 and 3.10.

Lemma 4.4. Let $X$ be the space of Example 4.1. If $x \in X$ and $N_{x}$ is any basic neighborhood of $x$, then $\mathrm{cl} N_{x} \backslash N_{x} \subseteq C \cup D$.

Proof. If $x \in A$, say $x=a_{p q}$ and $N_{x}=N_{k}\left(a_{p q}\right)$, then since $\phi$ is bijective, $N_{k}\left(a_{p q}\right) \cap N_{r}\left(a_{i j}\right)=\phi$ for all $i, j, r \in N$ such that $p \neq i$ and/or $q \neq j$. Also, $X \backslash T_{p, 1} \in \eta_{\infty}$ such that $N_{k}\left(a_{p q}\right) \cap X \backslash T_{p, 1} \subseteq N_{1}\left(c_{p}\right) \cap X \backslash T_{p, 1}=\phi$. Hence $\left(\operatorname{cl} N_{x} \backslash N_{x}\right) \cap(A \cup\{\infty\})=\phi$.

If $x \in C$, say $x=c_{i}$ and $N_{x}=N_{k}\left(c_{i}\right)$, then for $a_{p q} \in A$ either $a_{p q} \in$ $N_{k}\left(c_{i}\right)$ or $a_{p q} \notin N_{k}\left(c_{i}\right)$ so that either $N_{k}\left(a_{p q}\right) \subseteq N_{k}\left(c_{i}\right)$ or, for $r \in N$, $N_{r}\left(a_{p q}\right) \subseteq X \backslash N_{k}\left(c_{i}\right)$. Also, $X \backslash T_{i, k} \in \eta_{\infty}$ such that $X \backslash T_{i, k} \subseteq X \backslash N_{k}\left(c_{i}\right)$. Hence, $\left(\operatorname{cl} N_{x} \backslash N_{x}\right) \cap(A \cup\{\infty\})=\phi$.

If $x \in D$, say $x=d_{i}$ and $N_{x}=N_{k}\left(d_{i}\right)$, then since $\phi$ maps $A$ to $N \backslash\{1\}$, $N_{i+1}\left(a_{p q}\right) \cap N_{k}\left(d_{i}\right)=\phi$ for all $p, q \in N$. Also, $X \backslash T_{i, k} \in \eta_{\infty}$ such that $X \backslash T_{i, k} \subseteq$ $X \backslash N_{k}\left(d_{i}\right)$. Hence, $\left(\operatorname{cl} N_{x} \backslash N_{x}\right) \cap(A \cup\{\infty\})=\phi$.

If $x=\infty$ and $N_{\infty}=X \backslash T_{k, r}$, let $a_{p q}$ be an element of $A$. Either $a_{p q} \in$ $N_{r}\left(c_{i}\right)$ for some $i \in\{1,2, \ldots, k\}$ and therefore $N_{r}\left(a_{p q}\right) \subseteq N_{r}\left(c_{i}\right) \subseteq T_{k, r} \subseteq$ $X \backslash N_{\infty}$, or $a_{p q} \in A \cap\left(\bigcap_{i=1}^{k}\left[X \backslash N_{r}\left(c_{i}\right)\right]\right) \subseteq A \cap\left(X \backslash \bigcup_{i=1}^{k}\left[N_{r}\left(c_{i}\right) \cup N_{r}\left(d_{i}\right)\right]\right) \subseteq$ $X \backslash T_{k, r}=N_{\infty}$. Hence $\left(\operatorname{cl} N_{x} \backslash N_{x}\right) \cap(A \cup\{\infty\})=\phi$.

Since $B$ is discrete, if $b_{p q} \in B$, then $b_{p q} \notin \operatorname{cl} N_{x} \backslash N_{x}$ for every $x \in X$ and $N_{x} \in \eta_{x}$.

Thus if $x \in X$ and $N_{x}$ is any basic neighborhood of $x$, then $\left(\operatorname{cl} N_{x} \backslash N_{x}\right) \cap$ $(A \cup B \cup\{\infty\})=\phi$. This establishes the lemma.

Proposition 4.5. The space $X$ of Example 4.1 is adherent compact.

Proof. Since $X$ is $H$-closed, it suffices to show that every closed subset of $X$ is an open filter adherence. To this end, let $S$ be any closed subset of $X$ and assume that $S$ is not an open filter adherence. Then, given any cover $\left\{N_{x} \mid x \in \mathrm{cl}_{\Theta} S \backslash S\right\}$ of $\mathrm{cl}_{\Theta} S \backslash S$ consisting of basic neighborhoods contained in $X \backslash S$, there exists a finite subset $\left\{x_{1}, x_{2}, \ldots, x_{n}\right\} \subseteq \operatorname{cl}_{\Theta} S \backslash S$ such that $\phi \neq$ $\operatorname{int}\left(\bigcup_{t=1}^{n} \operatorname{cl} N_{x_{t}}\right) \cap S \subseteq\left(\bigcup_{t=1}^{n} \operatorname{cl} N_{x_{t}}\right) \cap S=\left[\bigcup_{t=1}^{n}\left(\operatorname{cl} N_{x_{t}} \backslash N_{x_{t}}\right)\right] \cap S \subseteq(C \cup D) \cap S$ by Lemma 4.4. If $C \cap S \neq \phi$, let $c_{j} \in C \cap S$; and if $D \cap S \neq \phi$, let $d_{i} \in D \cap S$. Let $m=\max \{i, j\}$ and let $N_{\infty} \equiv\left(X \backslash T_{m, 1}\right) \cap(X \backslash S)$. (If $C \cap S=\phi$ take $m=i$; and if $D \cap S=\phi$, take $m=j$.) For $x \in \mathrm{cl}_{\Theta} S \backslash(S \cup\{\infty\})$, let $N_{k}(x)$ be any basic neighborhood of $x$ contained in $X \backslash S$. It will be shown that the cover $\left\{N_{\infty}\right\} \cup\left\{N_{k}(x) \mid x \in \mathrm{cl}_{\Theta} S \backslash(S \cup\{\infty\})\right\}$ of $\mathrm{cl}_{\Theta} S \backslash S$ has the property: if $\left\{x_{1}, x_{2}, \ldots, x_{n}\right\} \subseteq \mathrm{cl}_{\Theta} S \backslash S$, then int $\left[\bigcup_{t=1}^{n} \operatorname{cl} N_{k}\left(x_{t}\right)\right] \cap S=\phi$.

Let $\left\{x_{2}, x_{2}, \ldots, x_{n}\right\} \subseteq \mathrm{cl}_{\Theta} S \backslash S$ and suppose that $c_{p}, d_{r} \in \operatorname{int}\left[\bigcup_{t=1}^{n} \operatorname{cl} N_{k}\left(x_{t}\right)\right]$ $\cap S$. Then $\operatorname{int}\left[\bigcup_{t=1}^{n} \mathrm{cl} N_{k}\left(x_{t}\right)\right]$ contains some basic neighborhoods of $c_{p}$ and $d_{r}$, and therefore contains $\left\{a_{p q} \mid q \in N_{j}, q \geq l\right\}$ and $\left\{b_{r q} \mid q \in N_{1}^{i}, q \geq l\right\}$ for 
some $l \in N$. However, given $t \in\{1,2, \ldots, n\}$ :

if $x_{t}=a_{v w} \in A$, then $\operatorname{cl} N_{k}\left(x_{t}\right) \cap\left\{a_{p q} \mid q \in N_{j}, q \geq l\right\} \subseteq\left\{a_{v w}\right\}$
and $\operatorname{cl} N_{k}\left(X_{t}\right) \cap\left\{b_{r q} \mid q \in N_{1}^{i}, q \geq l\right\} \subseteq\left\{b_{s q} \mid s \geq k, q \in N_{\phi\left(a_{v w}\right)}\right\} \cap$
$\left\{b_{r q} \mid q \in N_{1}^{i}, q \geq l\right\}=\phi ;$ if $x_{t}=c_{v} \in C$, then $c_{v} \notin\left\{c_{j}, c_{p}\right\}$
so that $\operatorname{cl} N_{k}\left(x_{t}\right) \cap\left\{a_{p q} \mid q \in N_{j}, q \geq l\right\} \subseteq\left[\left\{a_{v q} \mid q \geq k\right\} \cup\left\{a_{s q} \mid s \geq\right.\right.$
$\left.\left.k, q \in N_{v}\right\}\right] \cap\left\{a_{p q} \mid q \in N_{j}, q \geq l\right\}=\phi$ and $\operatorname{cl} N_{k}\left(x_{t}\right) \cap\left\{b_{r q} \mid q \in\right.$
$\left.N_{1}^{i}, q \geq l\right\} \subseteq\left\{b_{s q} \mid s \geq k, q \in \cup_{m>1} N_{m}\right\} \cap\left\{b_{r q} \mid q \in N_{1}^{i}, q \geq\right.$
$l\}=\phi ;$ and if $x_{t}=d_{v} \in D$, then $d_{v} \notin\left\{d_{i}, d_{r}\right\}$ so that
$\operatorname{cl} N_{k}\left(x_{t}\right) \cap\left\{a_{p q} \mid q \in N_{j}, q \geq l\right\}=\phi$ and $\operatorname{cl} N_{k}\left(x_{t}\right) \cap\left\{b_{r q} \mid q \in\right.$
$\left.N_{1}^{i}, q \geq l\right\} \subseteq\left[\left\{b_{v q} \mid q \geq k\right\} \cup\left\{b_{s q} \mid s \geq k, q \in N_{1}^{v}\right\}\right] \cap\left\{b_{r q} \mid q \in\right.$
$\left.N_{1}^{i}, q \geq l\right\}=\phi$.

Since $\operatorname{cl} N_{\infty} \cap\left[\left\{a_{p q} \mid q \in N_{j}, q \geq l\right\} \cup\left\{b_{r q} \mid q \in N_{1}^{i}, q \geq l\right\}\right] \subseteq\left(X \backslash\left[\operatorname{cl} N_{1}\left(c_{j}\right) \cup\right.\right.$ $\left.\left.\operatorname{cl} N_{1}\left(d_{i}\right)\right]\right) \cap\left[\left\{a_{p q} \mid q \in N_{j}\right\} \cup\left\{b_{r q} \mid q \in N_{1}^{i}\right\}\right] \subseteq\left(X \backslash\left[\operatorname{cl} N_{1}\left(c_{j}\right) \cup \operatorname{cl} N_{1}\left(d_{i}\right)\right]\right) \cap\left[N_{1}\left(c_{j}\right) \cup\right.$ $\left.N_{1}\left(d_{i}\right)\right]=\phi$, it follows that $\left[\bigcup_{t=1}^{n} \operatorname{cl} N_{k}\left(x_{t}\right)\right] \cap\left[\left\{a_{p q} \mid q \in N_{j}, q \geq l\right\} \cup\left\{b_{r q} \mid q \in\right.\right.$ $\left.\left.N_{1}^{i}, q \geq l\right\}\right] \subseteq\left\{x_{1}, x_{2}, \ldots, x_{n}\right\}$. This is a contradiction since $\left\{a_{p q} \mid q \in N_{j}, q \geq\right.$ l\} and $\left\{b_{r q} \mid q \in N_{1}^{i}, q \geq l\right\}$ are both infinite and were assumed to be contained in $\bigcup_{t=1}^{n} \operatorname{cl} N_{k}\left(x_{t}\right)$. Thus every closed subset of $X$ is an open filter adherence; and therefore, $X$ is adherent compact.

Remark 4.6. The space $X$ of Example 4.1 was the first space recognized by the author as being adherent compact but not seminormal. It should be noted, however, that the construction of Example 3.4 will yield a nonseminormal adherent compact space if each $X_{n}, n \in N^{*}$, is taken to be a copy of $X_{k}$ where $k$ is any fixed element of $N$. The closed subset $\{\beta\} \cup\left\{a^{i} \mid i \in N\right\}$ of the resulting space will have no regular open neighborhood contained in the complement of $\bigcup_{n \in N} C_{n}$. It should also be noted that the space of Example 3.1 is both $H$-closed seminormal and adherent compact. The author is unaware of any $H$-closed seminormal spaces which are not adherent compact.

\section{ACKNOWLEDGMENT}

The author wishes to thank the referees, and C. T. Scarborough, for their many helpful suggestions.

\section{BIBLIOGRAPHY}

1. A. Bella, On the number of $H$-sets in a Hausdorff space, (Italian Summary) Rend. Acad. Naz. Sci. XL Mem. Mat. 10 (5) (1986), no. 1, 251-254.

2. D. Burke and R. Hodel, The number of compact subsets of a topological space, Proc. Amer. Math. Soc. 58 (1976), 363-368.

3. S. Fomin, Extensions of topological spaces, Ann. Math. 44 (1943), 471-480.

4. G. Goss and G. Viglino, Some topological properties weaker than compactness, Pacific J. Math. 35 (1970), 635-638. 
5. __ C-compact and functionally compact spaces, Pacific J. Math. 37 (1971), 677-681.

6. M. Katětov, Über H-abgeschlossen und bikompact Räume, Časopis Pěst. Mat. Fys. 69 (1940), $36-49$.

7. R. Krystock, On H-sets and open filter adherences, Canad. Math. Bull. 31 (1988), 37-44.

8. C.-T. Liu, Absolutely closed spaces, Trans. Amer. Math. Soc. 130 (1968), 86-104.

9. J. Porter and M. Tikoo, On Katětov spaces, 1988 Spring Topology Conference, University of Florida.

10. M. H. Stone, Applications of the theory of Boolean rings to General Topology, Trans. Amer. Math. Soc. 41 (1937), 375-481.

11. G. Viglino, C-compact spaces, Duke J. Math. 36 (1969), 761-764.

12. __ Seminormal and C-compact spaces, Duke J. Math. 38 (1971), 57-61.

13. S. Willard, General Topology, Addison-Wesley, Reading, Massachusetts, 1970.

Mississippi State University, Mississippi State, Mississippi 39762 